OPEN ACCESS

Edited by:

David F. Clayton,

Clemson University, United States

Reviewed by:

Rossen Donev,

MicroPharm Ltd., United Kingdom

Claus Jürgen Scholz,

Labor Dr. Wisplinghoff, Germany

${ }^{*}$ Correspondence:

Min Hou

minhou@sjtu.edu.cn;

min-hou710@hotmail.com

Hui Wang

huiwang@shsmu.edu.cn

tThese authors have contributed equally to this work

Specialty section:

This article was submitted to

Neurogenomics,

a section of the journal

Frontiers in Neuroscience

Received: 19 October 2020

Accepted: 14 January 2021

Published: 24 February 2021

Citation:

Hou M, Xu G, Ran M, Luo W and

Wang $H$ (2021) APOE- $\varepsilon 4$ Carrier Status and Gut Microbiota Dysbiosis in Patients With Alzheimer Disease.

Front. Neurosci. 15:619051.

doi: 10.3389/fnins.2021.619051

\section{APOE- 44 Carrier Status and Gut Microbiota Dysbiosis in Patients With Alzheimer Disease}

\author{
Min $\mathrm{Hou}^{1 * \dagger}$, Gaolian Xü, Maosheng Ran ${ }^{2+}$, Wei Luo ${ }^{4}$ and Hui Wang ${ }^{1,5 *}$ \\ 'School of Public Health, College of Medicine, Shanghai Jiao Tong University, Shanghai, China, ${ }^{2}$ Nano Biomedical Research \\ Center, School of Biomedical Engineering, Shanghai Jiao Tong University, Shanghai, China, ${ }^{3}$ Department of Social Work \\ and Social Administration, The University of Hong Kong, Hong Kong, China, ${ }^{4}$ Xinjin No. 2 People's Hospital, Chengdu, \\ China, ${ }^{5}$ Center for Single-Cell Omics, School of Public Health, Shanghai Jiao Tong University School of Medicine, Shanghai, \\ China
}

Background: Alternations in gut microbiota and a number of genes have been implicated as risk factors for the development of Alzheimer disease (AD). However, the interactions between the altered bacteria and risk genetic variants remain unclear.

Objective: We aimed to explore associations of the risk genetic variants with altered gut bacteria in the onset of $A D$.

Methods: We collected baseline data and stool and blood samples from $30 \mathrm{AD}$ patients and 47 healthy controls in a case-control study. The rs42358/rs4512 (ApoE), rs3851179 (PICALM), rs744373 (BIN1), rs9331888 (CLU), rs670139 (MS4A4E), rs3764650 (ABCA7), rs3865444 (CD33), rs9349407 (CD2AP), rs11771145 (EPHA1), and rs3818361/rs6656401 (CR1) were sequenced, and microbiota composition was characterized using 16S rRNA gene sequencing. The associations of the altered gut bacteria with the risk genetics were analyzed.

Results: Apolipoprotein $\varepsilon 4$ allele and rs744373 were risk loci for the AD among 12 genetic variants. Phylum Proteobacteria; orders Enterobacteriales, Deltaproteobacteria, and Desulfovibrionales; families Enterobacteriaceae and Desulfovibrionaceae; and genera Escherichia-Shigella, Ruminococcaceae_UCG_002, Shuttleworthia, Anaerofustis, Morganelia, Finegoldia, and Anaerotruncus were increased in AD subjects, whereas family Enterococcaceae and genera Megamonas, Enterococcus, and Anaerostipes were more abundant in controls $(P<0.05)$. Among the altered microbiota, $\mathrm{APOE} \varepsilon 4$ allele was positively associated with pathogens: Proteobacteria.

Conclusion: The interaction of APOE $\varepsilon 4$ gene and the AD-promoting pathogens might be an important factor requiring for the promotion of $A D$. Targeting to microbiota might be an effective therapeutic strategy for AD susceptible to APOE $\varepsilon 4$ allele. This needs further investigation.

Keywords: Alzheimer disease, apolipoprotein E, dysbiosis, genetic variants, gut microbiome 


\section{INTRODUCTION}

Alzheimer disease (AD) is the most common form of dementia (Masters et al., 2015). More than 47 million people are currently afflicted worldwide, with this number predicted to reach 131.5 million by the year 2050 (Bahar-Fuchs et al., 2019). By 2010, 5.69 million Chinese people were living with $\mathrm{AD}$, a 3-fold increase from the previous decade (Clay et al., 2019), which have been partially attributed to an increasing elderly population. Symptoms include a progressive and global deterioration in memory, learning, orientation, language, comprehension, and judgment (Nussbaum and Ellis, 2003). The main pathology of AD involves a higher level of extracellular amyloid-beta $(A \beta)$ peptide in brain tissue, depositing in diffuse and neuritic plaques, and intracellular hyperphosphorylated tau (a microtubule assembly protein, p-tau) accumulating as neurofibrillary tangles (Dubois et al., 2016). However, pathogenesis has not been fully elucidated. Moreover, no treatments or interventions have been found to date that can effectively mitigate the progression of AD.

Risk factors for the development of $\mathrm{AD}$ have been identified in previous publications and include a genetic predisposition (Moustafa et al., 2018) and environmental factors (Daviglus et al., 2010; Barnes and Yaffe, 2011). Genome-wide association studies have identified polymorphisms with various levels of risk to develop AD, such as ABCA7, BIN1, CASS4, CD33, CD2AP, CELF1, CLU, CR1, DSG2, EPHA1, FERMT2, HLA-DRB5-DBR1, INPP5D, MS4A, MEF2C, NME8, PICALM, PTK2B, SLC24H4RIN3, SORL1, and ZCWPW1 (Harold et al., 2009; Lambert et al., 2009; Moustafa et al., 2018). Particularly, the greatest genetic risk factor is apolipoprotein E (APOE) genotype, with the presence of a single $\varepsilon 4$ allele increasing the risk by 3 - to 4 -fold compared with $\varepsilon 2$ or $\varepsilon 3$ allele (Corder et al., 1993). The APOE functions to transport cholesterol and other lipids to cells, facilitate their cellular uptake (Mahley, 1988), and to promote $A \beta$ clearance and neuronal signaling (Herz and Beffert, 2000). However, the specific functions of APOE that are associated with the development of $\mathrm{AD}$ remain unclear.

Recent evidence also implicates a role of the gut microbiome in the development of AD (Bonfili et al., 2017; Vogt et al., 2017; Zhang et al., 2017; Zhuang et al., 2018; Liu et al., 2019; Saji et al., 2019; Kim et al., 2020). The human gut microbiome consists of approximately $10^{14}$ microbes, 10 times the number of cells present in host, and is dominated by Firmicutes (60$80 \%$ ) and Bacteroidetes (20-30\%) species (Ley et al., 2006; Consortium, 2012). The gut microbiome in patients with $\mathrm{AD}$ has been identified as distinct in composition compared with subjects without cognitive impairment. For example, Bifidobacteria are reduced (Vogt et al., 2017), whereas Lachnospiraceae (Vogt et al., 2017), Gammaproteobacteria, Enterobacteriales, and Enterobacteriaceae species have been shown to be increased (Liu et al., 2019).

It has been suggested that the gut microbiome regulates multiple neurochemical pathways (Bonfili et al., 2017; Sun et al., 2020). The gram-positive bacteria Lactobacillus brevis and Bifidobacterium dentium enable the production of $\gamma$-aminobutyric acid (GABA), a major inhibitory neurotransmitter in human central nervous system (CNS)
(Barrett et al., 2012), and lower concentrations of GABA have been found in the frontal, temporal, and parietal cortex of patients with $\mathrm{AD}$ compared with individuals without $\mathrm{AD}$ (Lanctot et al., 2004). Inversely, the neurotoxins saxitoxin and anatoxin- $\alpha$, produced by various Cyanobacteria, have been shown to contribute to $\mathrm{AD}$ progression (Brenner, 2013). Moreover, gut dysbiosis may increase the levels of undesirable microbial metabolites in brain tissues such as lipopolysaccharides as a result of increased permeability of both the intestinal and the blood-brain barrier (Quigley, 2017). Current knowledge asserts that multiple factors shape the gut microbiome, including age, genetics, and the diet of the host (Benson et al., 2010). However, few studies have investigated factors impacting gut bacteria that were different between individuals with $\mathrm{AD}$ and without $\mathrm{AD}$.

Several recent studies have demonstrated that specific genetic loci contribute to alterations in gut microbial composition, such as HLA genes for autoimmunity (Russell et al., 2019), NOD2 for inflammatory bowel disease (Aschard et al., 2019), and MUC19 for primary sclerosing cholangitis (Eksteen, 2014). A recent study also evaluated associations between APOE genotypes and the abundance of Prevotellaceae, Ruminococcaceae, and several butyrate-producing species in healthy humans aged between 56 and 78 years (Tran et al., 2019). However, this has not yet been investigated in other populations, especially in those with $\mathrm{AD}$. Thus, the extent to which human genetics related to $\mathrm{AD}$ that shape microbiome composition, especially specific gut microbes for $\mathrm{AD}$, remains unclear. Thus, in this study, we explored genetic factors to $\mathrm{AD}$ and gut bacteria that were different between patients with AD compared with cognitive healthy people, as well as the effects of identified genetic variants on the bacteria.

\section{MATERIALS AND METHODS}

\section{Study Population}

Participants were recruited from Xinjin, Chengdu, China. Two hundred fifty-two subjects were diagnosed with dementia due to $\mathrm{AD}$, after retrieving the medical records from community hospitals and an epidemiological survey on mental health by a multidisciplinary team of neurologists, neuropsychologists, sociologists, and nurses in 2015. The diagnosis of AD accorded with the Diagnostic and Statistical Manual of Mental Disorders, Third Edition, revised, dementia criteria; the National Institute of Neurological and Communicative Disorders and Stroke; and the Alzheimer Disease and Related Disorders Association criteria, including both possible and probable AD (McKhann et al., 1984). In the end, 105 patients with $\mathrm{AD}$ and 554 healthy participants completed questionnaires by caregivers or themselves in 2018 . Since then, these participants will be seen every 3 years, and annually for 80 years. These questionnaires were repeated in the follow-up inquiries.

As the effects of age and gender on $\mathrm{AD}$ incidence are welldocumented (Fratiglioni et al., 1991; Vermunt et al., 2019), these factors were used as matching variables in this study. Controls were matched on age (within 5 years) and sex. If we were not able to find suitable controls for all $\mathrm{AD}$ cases using the matching criteria, two more matching cycles were performed. Criteria were 
relaxed in each cycle to obtain more matches. If there were not enough controls, stratified random sampling based on age and sex was used to maintain overall similarities between AD patients and controls. There were 30 patients with AD included in the final study aged 60-80 years, and there were 47 subjects without cognitive impairment; their gut microbiota speciation and polymorphisms were analyzed. Individuals included as controls underwent an assessment and were examined as having normal cognition. A screening questionnaire was provided to subjects for exclusion including history of diseases, use of medicine, antibiotics, nutritional supplements, and questions related to diet. Exclusion criteria for this study included any significant neurologic disease, gastrointestinal disease, chronic constipation, Clostridium difficile infection, history of alcohol/substance dependence, major psychiatric disorders, or any cancers. Those who have received antibiotics for at least 3 months prior to sampling, have any eating disorder; have dietary change for at least 1 month; were on any nutritional supplement such as probiotics or special diet; and have history of gastrointestinal operations that could confound with the results of gut microbiota were excluded for fecal collection. All participants or family caregivers provided written informed consent before involvement in this study. The Ethics Committee of School of Public Health at Shanghai Jiao Tong University for Human Subject Research approved all study procedures, and all experiments were performed in accordance with relevant guidelines and regulations.

\section{Clinical Information}

On the morning of blood sample collection, pulse and blood pressure were measured using a validated digital electronic device (HEM-7080IC; Omron Healthcare, Kyoto, Japan) by public health nurses. Measurement of fasting glucose, serum creatinine, total cholesterol, high-density lipoprotein (HDL) cholesterol, low-density lipoprotein (LDL) cholesterol, and triglycerides were obtained from the latest clinical records within 1 year.

\section{Stool Sample Collection and Fecal DNA Extraction}

Fecal samples were obtained from participants by family caregivers or themselves at home and immediately returned by delivery sample collection kits, packaged within insulated containers, and chilled with frozen gel packs for transportations to community hospitals. Finally, fecal samples were successfully collected from 21 patients with $\mathrm{AD}$ and 40 controls. All fecal samples were processed on the day of fecal collections. Upon receipt, chilled samples were weighed, and $200 \mathrm{mg}$ of aliquots was prepared into sterile bead beating tubes and remained frozen at $-80^{\circ} \mathrm{C}$ until DNA isolation. Full details of the DNA extraction are in online Supplementary Material.

\section{V3-V4 16S Sequencing Using Illumina MiSeq $2 \times 300$ bp}

Illumina MiSeq system was used to generate nucleotidesequencing data for 61 samples with 436 of sequencing average length. Sequencing read preprocessing, including merging, and demultiplexing, was done. Additional details are shown in the Supplementary Material.

\section{Operational Taxonomic Units Picking and Filtering}

The sequences were then clustered into operational taxonomic units (OTUs) using UPARSE (version 7.1) ${ }^{1}$ with a novel "greedy" algorithm with $97 \%$ similarity and taxonomically classified using the Greengenes 13.5 reference database (McDonald et al., 2012). Additional details are in the Supplementary Material.

\section{Single-Nucleotide Polymorphism Selection and Genotyping}

Blood $(6 \mathrm{ml})$ was obtained from participants in the morning using EDTA blood-collecting tube in each community hospitals after the $12 \mathrm{~h}$ fasting. The $0.5 \mathrm{ml}$ of whole blood was separated in the cold and frozen at $-80^{\circ} \mathrm{C}$ until DNA extraction. DNA was extracted from whole blood with the use of the Qiagen DNA blood kit (Qiagen, Germantown, MD, United States). The remaining blood sample was separated from by centrifugation at $3000 \times g$ for $15 \mathrm{~min}$ at $4^{\circ} \mathrm{C}$, and plasma was collected and stored at $-80^{\circ} \mathrm{C}$ for other analysis.

\section{DNA Purification and Quality Control Procedures}

Total twelve reported AD genetic risk variants were selected for the single-nucleotide polymorphism (SNP)-microbiota interaction analyses including rs42358 (ApoE), rs4512 (ApoE), rs3851179 (PICALM), rs744373 (BIN1), rs9331888 (CLU), rs670139 (MS4A4E), rs3764650 (ABCA7), rs3865444 (CD33), rs9349407 (CD2AP), and rs11771145 (EPHA1) and genetic variants in CR1: rs3818361 and rs6656401. We selected these risk variants ensuring that the selected genetic risk SNPs are functional variants or are in strong linkage disequilibrium with functional variants (Bradshaw et al., 2013; Imhann et al., 2018) to explore the interaction of the host with the gut microbiota. The DNA fragments containing the SNP sites including rs3851179 (PICALM), rs11771145 (EPHA1), rs3818361 (CR1), rs6656401 (CR1), rs7412 (APOE), and rs429358 (APOE) were amplified by polymerase chain reaction (PCR) with specific primers (Supplementary Table 1). The PCR products of these fragments were directly sequenced using the PCR amplification primers (Germer et al., 2000) with the data shown in online Supplementary Figure 1. Other AD genetic risk variants included rs744373 (BIN1), rs9331888 (CLU), rs670139 (MS4A4E), rs3764650 (ABCA7), rs3865444 (CD33), and rs9349407 (CD2AP), with the genotype tested using with allele-specific TaqMan assays in LightCycler ${ }^{\circledR} 480$ Instrument II (Roche Life Science) with 96-Well Block Module. The PCR primers and TaqMan probes are listed in online Supplementary Table 1. The genotype data were analyzed using the LightCycler software version 1.1. The APOE haplotypes $(\varepsilon 2 / \varepsilon 3, \varepsilon 3 / \varepsilon 3, \varepsilon 3 / \varepsilon 4$, and $\varepsilon 4 / \varepsilon 4$ ) were derived from the allelic combinations of the APOE SNPs rs7412 and rs429358.

\footnotetext{
${ }^{1}$ http://drive5.com/uparse/
} 


\section{Statistical Analysis}

Sample size for this study was determined using QUANTO version 1.2.4 ${ }^{2}$. As the importantly strongest genetic risk factor for $\mathrm{AD}$, assuming minor allele frequencies (MAFs) from 0.1, this study sample size of 77 cases is suitable to detect an allelic odds ratio (OR) of 4.5 at $80 \%$ statistical power and $5 \%$ significance level.

The continuous variables of baseline characteristics presented in this study were expressed as the means $\pm \mathrm{SD}$ or medians with interquartile range (IQR). And the categorical variables were expressed as frequencies and percentages. The normal distribution of variables including age, body mass index (BMI), waist, pulse, systolic blood pressure, diastolic blood pressure, fasting glucose, serum creatinine, total cholesterol, HDL cholesterol, LDL cholesterol, and triglycerides was assessed using the Shapiro-Wilk test. Baseline characteristics between patients with $\mathrm{AD}$ and controls were compared, using $\chi^{2}$, Fisher exact test, Wilcoxon-Mann-Whitney test, or Student $t$-test, where appropriate. $P<0.05$ was considered statistically significant. Analyses were completed using the IBM SPSS program, version 22 (IBM, Chicago, IL, United States).

\section{SNPs and APOE Genotype Analysis}

Analyses of genetic sequencing data were completed using the IBM SPSS program mentioned previously. SNPs with HardyWeinberg equilibrium test $p<0.05$ or MAF $<1 \%$ were excluded. A $\chi^{2}$ test was used to estimate the differences between the frequencies of the genotypes and alleles in patients with $\mathrm{AD}$ and the control group. Differences were considered significant at $P<0.05$.

Because certain APOE genotypes are relatively rare (e.g., $\varepsilon 2 / \varepsilon 2$ and $\varepsilon 4 / \varepsilon 4$ ), the APOE genotype ORs were calculated by dividing subjects into two main categories: those with at least one $\varepsilon 4$ allele present and those with no $\varepsilon 4$ allele. The associations between the candidate SNPs, APOE $\varepsilon 4$ allele and AD were estimated using ORs with $95 \%$ confidence intervals in logistic regression models, which were adjusted by including significant covariates, mainly age, gender, and APOE genotype.

\section{Microbial Data Processing}

The sequence data of the gut microbiota was mainly analyzed using the QIIME (1.9.1) (Caporaso et al., 2010) and R packages (v3.2.0). Beta diversity distances were calculated using Bray-Curtis dissimilarity and weighted/unweighted-UniFrac represented in principal coordinate analyses (PCoA) at OTU level. To detect statistical differences in beta diversity metrics between the $\mathrm{AD}$ and control groups, the permutational multivariate analysis of variance in the vegan package was used. Statistically significant differences in the relative abundances of taxa from phylum to species were calculated by using the linear discriminant analysis effect size method (LEfSe) with nonparametric factorial Kruskal-Wallis rank-sum test. Taxa with linear discriminant analysis (LDA) results of more than three were considered statistically significantly enriched.

${ }^{2}$ https://preventivemedicine.usc.edu/download-quanto

\section{Quantitative Trait Locus Association Analysis}

To link microbial composition to genetic variation, abundance values of taxonomies were treated as quantitative traits. Multivariate analysis was performed using multivariate association with linear models (MaAsLin) (Morgan et al., 2012) to identify associations of specific gut microbial taxa at all taxonomic levels from kingdom to genus with $\mathrm{AD}$-related SNPs and genotypes. Associations with a Benjamini and Hochberg false discovery rate (FDR)-corrected $p$-value ( $q$-value) of $<0.05$ were considered to be significant. Gut microbiota differences among subgroups defined by $\mathrm{AD}$ were assessed by the Wilcoxon-Mann-Whitney tests.

\section{RESULTS}

\section{Characteristics of Patients With AD and Cognitive Healthy Controls}

As shown in Table 1, the mean subject ages were, respectively $(\mathrm{SD}=71.9 \pm 6.9)$ years and $(\mathrm{SD}=71.1 \pm 6.7)$ years. Meanwhile, $43.3 \%$ and $53.2 \%$ of the subjects were female in the $\mathrm{AD}$ group

TABLE 1 | Participant characteristics in this study $(n=77)$.

\begin{tabular}{|c|c|c|c|}
\hline & $\begin{array}{l}\text { Patients with } \\
\text { AD }(n=30)\end{array}$ & $\begin{array}{l}\text { Controls } \\
(n=47)\end{array}$ & $p$-value \\
\hline Age (years) & $71.9 \pm 6.9$ & $71.1 \pm 6.7$ & 0.966 \\
\hline \multicolumn{4}{|l|}{ Gender } \\
\hline Male & $17(56.7)$ & $22(46.8)$ & 0.402 \\
\hline Female & $13(43.3)$ & $25(53.2)$ & \\
\hline $\mathrm{BMI}\left(\mathrm{kg} / \mathrm{m}^{2}\right)$ & $23.74 \pm 4.77$ & $23.63 \pm 3.31$ & 0.994 \\
\hline Waist (cm) & $77.47 \pm 8.92$ & $79.33 \pm 9.06$ & 0.935 \\
\hline \multicolumn{4}{|l|}{ Medical history } \\
\hline Hypertension & $13(43.3)$ & $22(50)$ & 0.032 \\
\hline Type 2 diabetes & 7 (23.3) & $3(6.8)$ & 0.767 \\
\hline Cardiovascular disease & $4(13.3)$ & $0(0)$ & 0.011 \\
\hline Marriage & & & 0.321 \\
\hline Single & $0(0)$ & $3(6.8)$ & \\
\hline Married & $30(100.00)$ & 44 (93.2) & \\
\hline Education & & & 0.527 \\
\hline No & $8(26.7)$ & $9(19.1)$ & \\
\hline Primary school & $13(43.3)$ & $26(55.3)$ & \\
\hline $\begin{array}{l}\text { Secondary high school and higher } \\
\text { level }\end{array}$ & $9(30.0)$ & $12(25.6)$ & \\
\hline Pulse & $75.00 \pm 12.42$ & $73.37 \pm 9.77$ & 0.440 \\
\hline Systolic blood pressure (mm Hg) & $131.84 \pm 14.59$ & $138.61 \pm 16.18$ & 0.436 \\
\hline Diastolic blood pressure $(\mathrm{mm} \mathrm{Hg})$ & $79.74 \pm 9.76$ & $80.63 \pm 9.64$ & 0.803 \\
\hline Fasting glucose (mmol/L) & $6.12 \pm 1.27$ & $5.47 \pm 1.06$ & 0.193 \\
\hline Serum creatinine ( $\mu \mathrm{mol} / \mathrm{L})$ & $71.51 \pm 37.31$ & $67.13 \pm 28.06$ & 0.165 \\
\hline Total cholesterol (mmol/L) & $4.56 \pm 0.90$ & $4.99 \pm 0.76$ & 0.310 \\
\hline HDL cholesterol (mmol/L) & $1.30 \pm 0.34$ & $1.46 \pm 0.67$ & 0.376 \\
\hline LDL cholesterol (mmol/L) & $2.57 \pm 0.71$ & $2.73 \pm 0.69$ & 0.603 \\
\hline Triglycerides (mmol/L) & $1.28 \pm 0.53$ & $1.49 \pm 0.68$ & 0.384 \\
\hline
\end{tabular}

Data are $n(\%)$, mean $\pm S D$, or median (IQR), unless otherwise specified. 
and control group, respectively, but not significantly different between groups $(p>0.05)$. In addition, the anthropometric characteristics including BMI, waist circumstance, pulse and blood pressure, and sociodemographic factors including educational level and marriage status were not significantly different between groups $(p>0.05)$. Moreover, the biochemical analyses including fasting glucose, total cholesterol, HDL cholesterol, LDL cholesterol, and triglycerides were not significantly different between groups $(p>0.05)$. There were $23.3 \%$ of $\mathrm{AD}$ patients with type 2 diabetes, but no significant difference compared with the control group $(p>0.05)$. The $43.3 \%$ of patients with $\mathrm{AD}$ had hypertension, and $13.3 \%$ of those had cardiovascular disease, and these numbers were significant different as compared to the healthy control group $(p=0.032 ; p=0.011)$.

\section{Genetic Variants and AD}

Using the $\chi^{2}$ test, the significant associations of $\mathrm{AD}$ status were found with APOE genotype and MS4A4E (rs670139), but not with PICALM (rs3851179), EPHA1 (rs11771145), CR1 (rs3818361), CR1 (rs6656401), CD33 (rs3865444), ABCA7 (rs3764650), CLU (rs9331888), BIN1 (rs744373), or CD2AP (rs9349407) (Table 2). Fifteen participants were APOE $\varepsilon 4$ carriers (APOE $\varepsilon 3 / \varepsilon 4$ and APOE $\varepsilon 4 / \varepsilon 4 ; 12$ men and 12 women), among whom 10 were patients with $\mathrm{AD}$. As expected, the frequency of APOE $\varepsilon 4$ carriers was significantly greater in patients with AD

TABLE 2 | The association of genetic variants with AD risk.

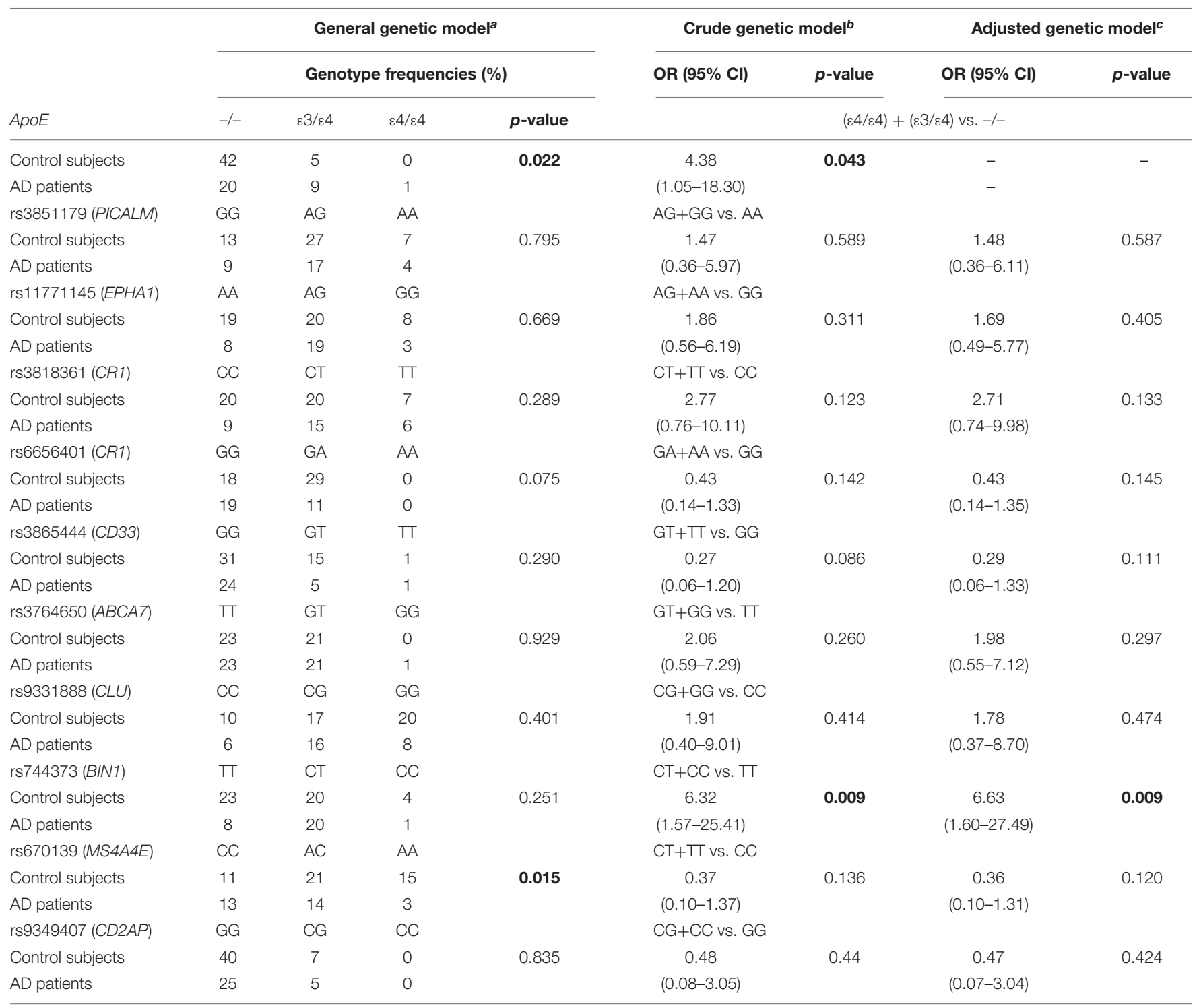

$P<0.05$ are in bold type.

${ }^{a} \chi^{2}$ test.

${ }^{b}$ Adjusted for age and gender.

${ }^{C}$ Adjusted for age, gender and APOE.

$\mathrm{Cl}$, confidence interval; OR, odds ratio; -/-, non- $\varepsilon 3$ or $-\varepsilon 4$. 
compared with normal cognitive individuals. The presence of at least one APOE $\varepsilon 4$ allele significantly increased the risk of $\mathrm{AD}$ compared with the absence of APOE $\varepsilon 4$ allele $(p=0.043$; OR, 4.38; 95\% CI, 1.05-18.30). In addition, harboring a $C$ allele in rs7744373 (BIN1) was associated with greater risk of $\mathrm{AD}$ compared with the homozygous TT genotype after adjustment for age and gender ( $p=0.009$; OR, 6.63; 95\% CI, 1.57-25.41).

\section{Subjects With AD Harbor the Altered Gut Microbiota}

To assess bacterial composition, we successfully collected 60 fecal samples, among which 21 subjects were patients with $\mathrm{AD}$ and 40 were cognitively healthy controls. In total, more than 4.5 million quality-filtered sequences were obtained $(49,274 \pm 11,495$ sequences per sample). We identified a total of 808 bacterial taxa across six hierarchical levels from phylum to species at 12,556 OTUs. The predominant phyla in both patients with $\mathrm{AD}$ and cognitive healthy controls were Firmicutes (52\% in patients with $\mathrm{AD}, 59 \%$ in cognitive healthy controls), Proteobacteria $(24 \%$ in patients with $\mathrm{AD}, 12 \%$ in cognitive healthy controls), and Bacteroidetes (18\% in patients with $\mathrm{AD}$, $22 \%$ in cognitive healthy controls). Clostridia was the most abundant class in patients with AD (42\%) and in healthy controls (49\%). Escherichia-Shigella was the most abundant genera in patients with $\mathrm{AD}(19 \%)$, whereas Bacteroides was the most abundant genus in healthy controls (12\%). Analysis of alpha diversity revealed no significant differences between $\mathrm{AD}$ and cognitive healthy subjects in the Sobs index $(p=0.056)$ and Shannon index $(p=0.687)$ using Wilcoxon rank-sum test as depicted in Figure 1. To test significant differences in the structure of gut microbiota responding to $\mathrm{AD}$ status, we next performed analysis of similarities on the $\beta$-diversity distances with unweighted/weighted UniFrac and Bray-Curtis distances. The PCoA analysis presented slight difference in gut microbial composition between groups calculated on the Bray-Curtis dissimilarity ( $p=0.039$ ), but hardly revealed distinguishable bacterial microbiota of AD subjects compared to cognitive healthy controls on the unweighted UniFrac $(p=0.065)$ and the weighted UniFrac ( $p=0.233$ ) (Figure 2).

The differences in the abundances of gut microbial taxa between $\mathrm{AD}$ patients and cognitive healthy subjects were analyzed using LEfSe with nonparametric factorial Kruskal-Wallis rank-sum test with the filtered set excluding low abundance $(<0.1 \%$ mean relative abundance) and $<3$. The results indicated 18 taxa were significantly altered in patients with AD from phylum to genera $(p<0.05)$ (Figure 3 ). Among those excluding unclassified, uncultured, and nonrank taxa, phylum Proteobacteria; orders Enterobacteriales,
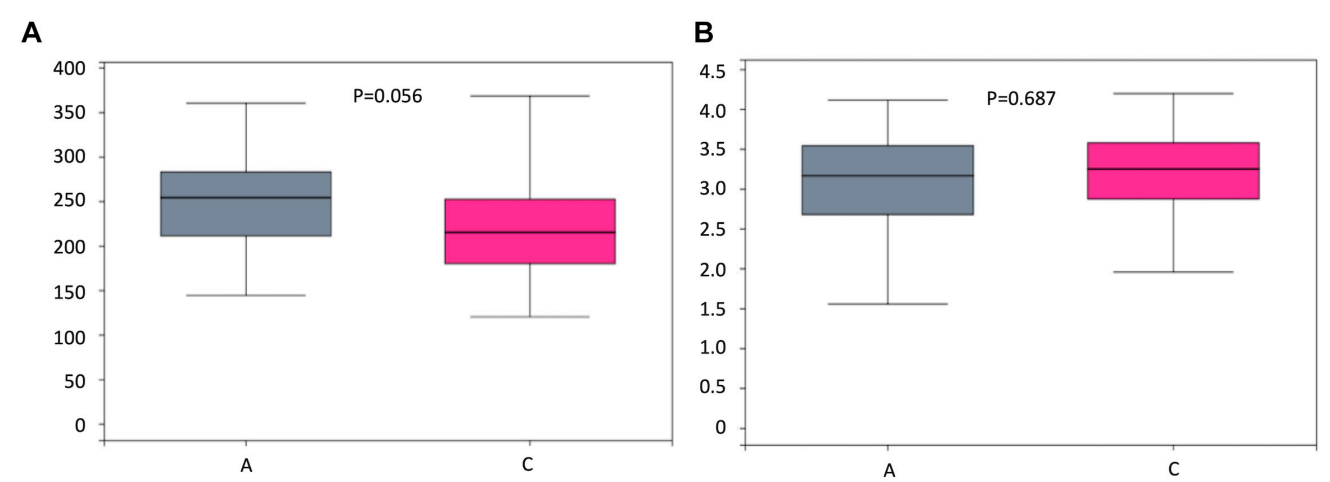

FIGURE 1 | Boxplots and statistical comparison (Wilcoxon rank-sum test) shows no significant difference between alpha diversities among groups including Sobs index (A) and Shannon index (B). P-values for each comparison are depicted above the boxplots of the groups being compared. Boxplot medians (center lines); interquartile ranges (box ranges). Patients with $A D$ and healthy subjects are, respectively, colored in gray and rose red.
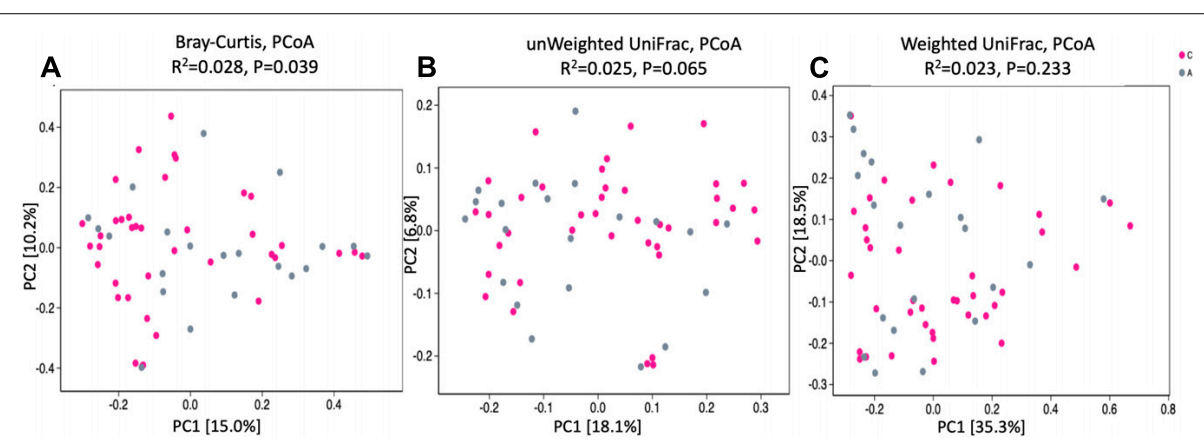

FIGURE 2 | PCoA of bacterial beta diversity based on (A) Bray-Curtis dissimilarity, (B) Unweighted, and (C) Weighted UniFrac distances. Patients with Alzheimer disease and healthy subjects are, respectively, colored in gray and rose red and are indicated by circles. 
Deltaproteobacteria, and Desulfovibrionales; families Enterobacteriaceae and Desulfovibrionaceae; genera EscherichiaShigella, Ruminococcaceae_UCG_002, Shuttleworthia, Anaerofustis, Morganelia, Finegoldia, and Anaerotruncus were increased in AD subjects, whereas family Enterococcaceae and genera Megamonas, Enterococcus, and Anaerostipes were more abundant in healthy controls $(P<0.05)$.

\section{Associations of Risk Genetic Variants With the Altered Gut Microbiota}

To investigate associations between two AD risk genetic variants in the gene BIN1 (rs744373) and APOE and specific microbial taxa related to $\mathrm{AD}$, the targeted analyses were performed using MaAsLin. Two significant associations were detected between APOE genotypes and AD-related gut microbial taxa. A higher number of the $\varepsilon 4$ allele was associated with an increase in the abundances of the phylum Proteobacteria (FDR$p=0.029$ ) and the family Enterococcaceae classified into the phylum Firmicutes (FDR- $p=0.046$ ) as depicted in Figure 4 (FDR $<0.05)$. The $\beta$-coefficients suggest that the associations of the APOE genotype with the phylum Firmicutes are of moderate effect size $(\beta=-0.23)$. In particular, bacteria displaying higher levels in carriers of $\mathrm{AD}$ genetic risk alleles are more likely to be positively associated with the risk of $\mathrm{AD}$. In addition, each additional copy of the minor A allele at nonAD-associated gene PICALM SNP rs3851179 increased the abundances of the Actinobacteria phylum with a light effect size $(\beta=-0.08, \mathrm{FDR}=0.034)$.

\section{DISCUSSION}

In this study, we explored genetic risks associated with $\mathrm{AD}$ and report that genetic variants including APOE genotype and BIN1 (rs744373) are significantly associated with abundance of specific gut microbiota. In particular, we found that the phylum Proteobacteria and the family Enterococcaceae are strongly associated with $\mathrm{AD}$ status, and their abundance is significantly increased in those subjects with an $A P O E \& 4$ genotype, leading to an increased risk of AD.

We found that both $A P O E \& 4$ genotype and an SNP in rs7744373 (BIN1) gene were significant risks for $\mathrm{AD}$ in a population after adjustment for age and gender, which is

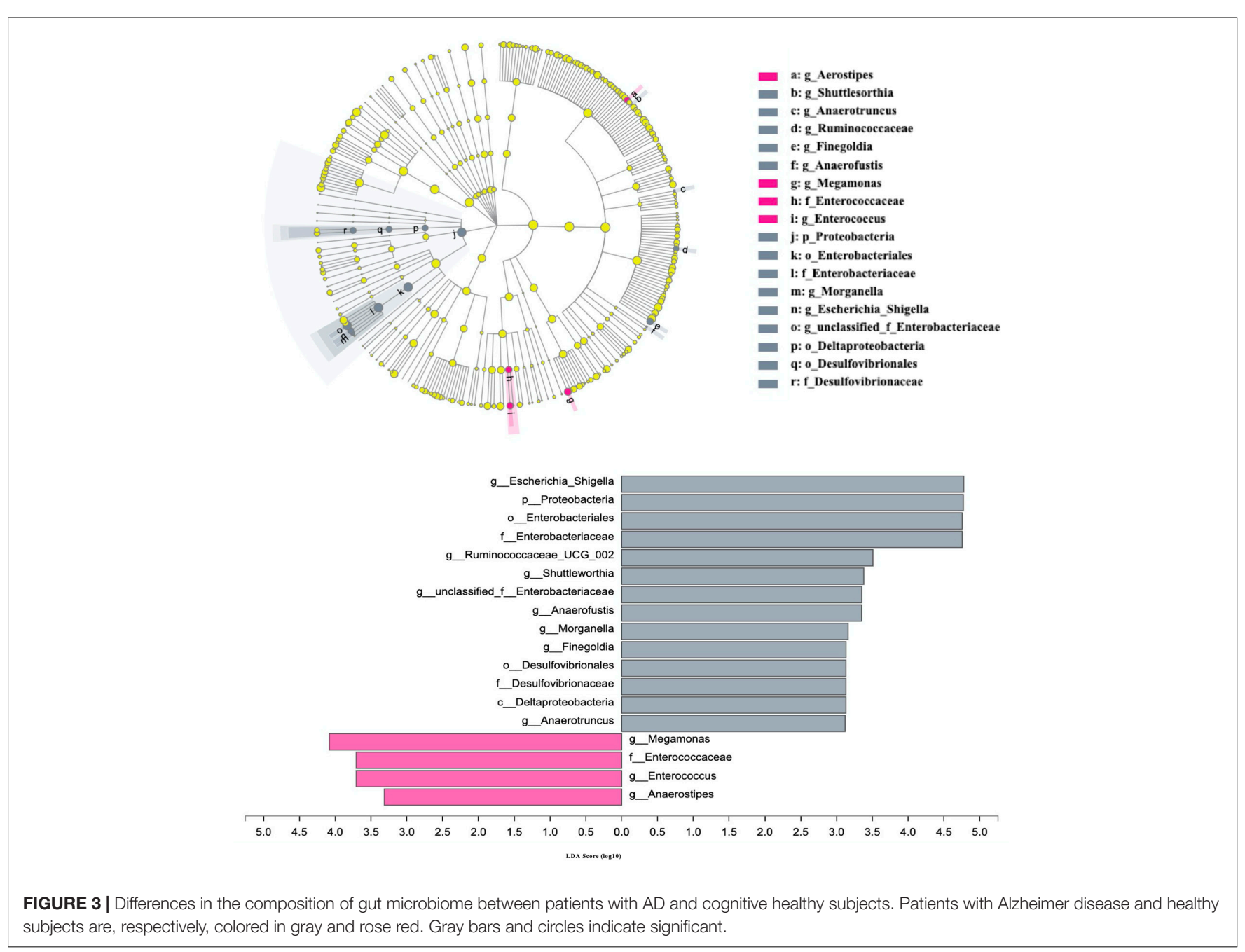



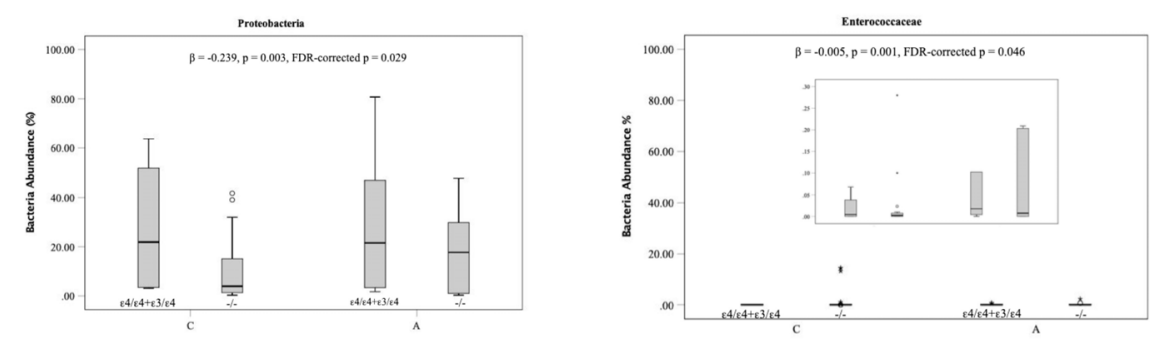

FIGURE 4 | Associations of $A D$ associated genetic variants with specific members of the gut microbiota. The boxplots indicate the median (horizontal solid line) and the IQR between the first and third quartiles (box). *indicate significance FDR $<0.05$. A, Alzheimer disease; C, Cognitive healthy subjects.

consistent with previous studies in Chinese populations (Tan et al., 2013; Han et al., 2019) and European-American subjects (Wijsman et al., 2011). After adjusting for APOE $\varepsilon 4$ genotype, the positive association with $\mathrm{AD}$ by $\mathrm{C}$ allele in rs7744373 (BIN1) remained. Previous studies indicate that BIN1 is expressed to regulate synaptic vesicle endocytosis and cytoskeletal dynamics (Cousin and Robinson, 2001), and its isoforms were different in multiple AD brain regions (Holler et al., 2014). It may act as a modulator of tangle pathology (Holler et al., 2014), but the exact function of this SNP still remains unknown.

Although a significant association with $\mathrm{AD}$ was not found in rs670139 (MS4A4E), the $p$-value for frequencies of the rs670139 alleles (CC:AC:AA) was significant $(p=0.015)$ in our analysis. Hence, further assessment of this SNP in a large sample with sufficient statistical power is needed.

The results indicated that 18 taxa were significantly altered in patients with $\mathrm{AD}$ from phylum to genera when compared to non-AD individuals $(p<0.05)$ (Figure 3). Decreased gut microbiota diversity has been reported in subjects with $\mathrm{AD}$ (Vogt et al., 2017; Liu et al., 2019). In this study, these 18 bacterial taxa characterized dysbiosis in the fecal microbiota of patients with $\mathrm{AD}$. The expansion of the phylum Proteobacteria in the microbial profiles of patients with $\mathrm{AD}$ was in agreement with recent findings of Liu et al. (2019) in patients with $\mathrm{AD}$ from a hospital in Hangzhou, East China. In addition, in this study, the increase of Enterobacteriaceae, a member of Proteobacteria, has been reported previously in patients with other CNS diseases, such as Parkinson disease (Unger et al., 2016) and major depressive disorder (Jiang et al., 2015). Interestingly, species Escherichia-Shigella and Morganelia as important pathogens belonging to Enterobacteriaceae (data not shown) were also enriched in patients with $\mathrm{AD}$ compared to healthy individuals and are catalase-positive and oxidativenegative in vitro (Adeolu et al., 2016). Importantly, we newly identified the reduction of genera Megamonas, Enterococcus, and Anaerostipes in patients with AD. Megamonas has been shown to produce short-chain fatty acids (SCFAs) derived from dietary fibers (Adeolu et al., 2016), which may benefit host energy metabolism to attenuate the development of $\mathrm{AD}$ (Ho et al., 2018). In addition, Anaerostipes, a member of family Lachnospiraceae, are also SCFA-producing bacteria (Schwiertz et al., 2002) and were detected at higher levels in non-AD Chinese rather than non-AD Caucasian as compared to $\mathrm{AD}$ subjects in previous works (Zhuang et al., 2018; Liu et al., 2019). This finding suggests that Anaerostipes may ethnically play a role in the development of AD. Furthermore, genus Enterococcus, a member of Lactobacillales, has been extremely studied as potential candidate probiotics by producing SCFAs for prevention of some human disease including irritable bowel syndrome symptoms (Avram-Hananel et al., 2010; Hanchi et al., 2018). Based on the results of this recent study and our findings, it would suggest that the reduction of SCFA-producing bacteria is involved in the development of the $\mathrm{AD}$. To our knowledge, the protective roles of SCFAs against the formation of toxic soluble $\beta$-amyloid aggregates (Ho et al., 2018) and in regulating microglial inflammatory response in vitro (Huuskonen et al., 2004) involved in the development of AD are well documented. Thus, these results provide an insight that gut microbiota alterations may contribute to or exacerbate $\mathrm{AD}$ pathology through modulation of host metabolism.

To assess associations of genetic risk loci with a number of bacterial taxa for $\mathrm{AD}$, we initially employed a considerably strict statistical cutoff ( $q<0.05$ in the MaAsLin) to avoid falsepositive results. To our knowledge, no such connections have been demonstrated to date in previous studies yet. In our present study, our finding has implications for an increase only in $\varepsilon 4$ allele of APOE genotype among 12 genetic variants involving the risk of the development of $\mathrm{AD}$ associated with the growth of the phylum Proteobacteria and family Enterococcaceae, independent of the individual's $\mathrm{AD}$ status. As mentioned previously, phylum Proteobacteria was more abundant in patient with $\mathrm{AD}$ as pathogens. Previous work noted in healthy humans and mice that $A P O E$ genotypes were significantly associated with the reduction of butyrate-producing bacteria that could promote health (Tran et al., 2019). Thus, expanding these previous hypotheses of the function of expressed apoE mentioned previously (Mahley, 1988; Herz and Beffert, 2000; Elliott et al., 2007), our findings add evidence that the ApoE genotype may have impact on the pathophysiology of AD through specific bacteria. However, the mechanism that shapes the gut microbiome by the ApoE genotype to promote $\mathrm{AD}$ pathogenesis will require a range of studies with knockout animal models to be fully understood.

Our study has several limitations. First, the samples with which we could evaluate this result were relatively small, but provided sufficient statistical power to detect relevant associations. Second, one single-center study may 
limit the application of the genetic and microbial interactions. However, subjects are ethnically and geographically homogenous individuals to minimize some confounders, such as the dietary factor. Future studies should be extended to the associations observed in multicenters of differing backgrounds. Third, rather than shotgun metagenomic sequencing of the entire DNA, we performed $16 \mathrm{~S}$ rRNA gene sequencing that limited data interpretation at species level and in functional information, but substantially broadened our understanding of overall bacterial structure and abundance in relation to AD. Moreover, the specific design of the study falls short of discerning the sequential causal relationship, which should be investigated in knockout animal models or in longitudinal studies before and after the onset of AD. Despite these limitations in this study, the results of ADassociated genetics and bacteria we found are in agreement with previous findings.

\section{CONCLUSION}

Our study demonstrated that Proteobacteria significantly increased in patients with $\mathrm{AD}$ status and had a relationship with genetic risk APOE genotype. This study provided an insight that changes in the gut microbiota were associated with specific host genetic variants, which is important for understanding the $\mathrm{AD}$ pathogenesis. Moreover, it is proposed that targeting to modulate gut microbiota, especially the improvement of SCFA-producing bacteria and reduction of pathogens, might be an effective therapeutic strategy for disorders susceptible to gene host genetics.

\section{DATA AVAILABILITY STATEMENT}

The datasets presented in this study can be found in online repositories. The names of the repository/repositories and accession number(s) can be found in the article/ Supplementary Material.

\section{ETHICS STATEMENT}

The studies involving human participants were reviewed and approved by the Ethics Committee of School of Public Health at

\section{REFERENCES}

Adeolu, M., Alnajar, S., Naushad, S., and Gupta, R. S. (2016). Genomebased phylogeny and taxonomy of the 'Enterobacteriales': proposal for Enterobacterales ord. nov. divided into the families Enterobacteriaceae, Erwiniaceae fam. nov., Pectobacteriaceae fam. nov., Yersiniaceae fam. nov., Hafniaceae fam. nov., Morganellaceae fam. nov., and Budviciaceae fam. nov. Int. J. Syst. Evol. Microbiol. 66, 5575-5599. doi: 10.1099/ijsem.0.001485

Aschard, H., Laville, V., Tchetgen, E. T., Knights, D., Imhann, F., Seksik, P., et al. (2019). Genetic effects on the commensal microbiota in inflammatory bowel disease patients. PLoS Genet. 15:e1008018. doi: 10.1371/journal.pgen.1008018

Avram-Hananel, L., Stock, J., Parlesak, A., Bode, C., and Schwartz, B. (2010). E durans strain M4-5 isolated from human colonic flora attenuates
Shanghai Jiao Tong University for Human Subject Research. The patients/participants provided their written informed consent to participate in this study.

\section{AUTHOR CONTRIBUTIONS}

MH, GX, and HW designed and conceptualized the study, and revised the manuscript. $\mathrm{MH}, \mathrm{MR}$, and $\mathrm{WL}$ contributed to data collection and database generation. $\mathrm{MH}$ and $\mathrm{GX}$ performed the experiments, data analysis, and interpretation. $\mathrm{MH}$ drafted and revised the manuscript. All authors read and approved the manuscript.

\section{FUNDING}

This work was supported by the Intersection of Arts and Science in Shanghai Jiao Tong University, 17JCYB12, Interdisciplinary Program of Shanghai Jiao Tong University, ZH2018QNA69, National Key R\&D Program of China, 2018YFC2000700, National Natural Science Foundation of China, 81630086, Key Research Program of the Chinese Academy of Sciences, ZDRWZS-2017-1, and Medicine and Engineering Interdisciplinary Research Fund of Shanghai Jiao Tong University, YG2020YQ06.

\section{ACKNOWLEDGMENTS}

We thank all participants in this study, and staffs in Xinjin No. 2 People's Hospital and health centers in Xinjin, Chengdu who collected samples and baseline data.

\section{SUPPLEMENTARY MATERIAL}

The Supplementary Material for this article can be found online at: https://www.frontiersin.org/articles/10.3389/fnins. 2021.619051/full\#supplementary-material

Supplementary Figure 1 | Individual PCR fragments.

Supplementary Table 1 | Primer information.

intestinal inflammation. Dis. Colon. Rectum 53, 1676-1686. doi: 10.1007/dcr. 0b013e3181f4b148

Bahar-Fuchs, A., Martyr, A., Goh, A. M., Sabates, J., and Clare, L. (2019). Cognitive training for people with mild to moderate dementia. Cochrane Database Syst. Rev. 3:Cd013069.

Barnes, D. E., and Yaffe, K. (2011). The projected effect of risk factor reduction on Alzheimer's disease prevalence. Lancet Neurol. 10, 819-828. doi: 10.1016/ s1474-4422(11)70072-2

Barrett, E., Ross, R. P., O’toole, P. W., Fitzgerald, G. F., and Stanton, C. (2012). gamma-Aminobutyric acid production by culturable bacteria from the human intestine. J. Appl. Microbiol. 113, 411-417. doi: 10.1111/j.1365-2672.2012. 05344.x

Benson, A. K., Kelly, S. A., Legge, R., Ma, F., Low, S. J., Kim, J., et al. (2010). Individuality in gut microbiota composition is a complex polygenic trait shaped by multiple environmental and host genetic factors. 
Proc. Natl. Acad. Sci. U.S.A. 107, 18933-18938. doi: 10.1073/pnas.100702 8107

Bonfili, L., Cecarini, V., Berardi, S., Scarpona, S., Suchodolski, J. S., Nasuti, C., et al. (2017). Microbiota modulation counteracts Alzheimer's disease progression influencing neuronal proteolysis and gut hormones plasma levels. Sci. Rep. $7: 2426$.

Bradshaw, E. M., Chibnik, L. B., Keenan, B. T., Ottoboni, L., Raj, T., Tang, A., et al. (2013). CD33 Alzheimer's disease locus: altered monocyte function and amyloid biology. Nat. Neurosci. 16, 848-850. doi: 10.1038/nn.3435

Brenner, S. R. (2013). Blue-green algae or cyanobacteria in the intestinal micro-flora may produce neurotoxins such as Beta-N-Methylamino-L-Alanine (BMAA) which may be related to development of amyotrophic lateral sclerosis, Alzheimer's disease and Parkinson-dementia-complex in humans and equine motor neuron disease in horses. Med. Hypotheses 80:103. doi: 10.1016/j.mehy. 2012.10.010

Caporaso, J. G., Kuczynski, J., Stombaugh, J., Bittinger, K., Bushman, F. D., Costello, E. K., et al. (2010). QIIME allows analysis of high-throughput community sequencing data. Nat. Methods 7, 335-336.

Clay, E., Zhou, J., Yi, Z. M., Zhai, S., and Toumi, M. (2019). Economic burden for Alzheimer's disease in China from 2010 to 2050: a modelling study. J. Mark. Access. Health Policy 7:1667195. doi: 10.1080/20016689.2019.1667195

Consortium, H. M. P. (2012). Structure, function and diversity of the healthy human microbiome. Nature 486, 207-214. doi: 10.1038/nature11234

Corder, E. H., Saunders, A. M., Strittmatter, W. J., Schmechel, D. E., Gaskell, P. C., Small, G. W., et al. (1993). Gene dose of apolipoprotein E type 4 allele and the risk of Alzheimer's disease in late onset families. Science 261, 921-923. doi: $10.1126 /$ science. 8346443

Cousin, M. A., and Robinson, P. J. (2001). The dephosphins: dephosphorylation by calcineurin triggers synaptic vesicle endocytosis. Trends Neurosci. 24, 659-665. doi: 10.1016/s0166-2236(00)01930-5

Daviglus, M. L., Bell, C. C., Berrettini, W., Bowen, P. E., Connolly, E. S. Jr., et al. (2010). National Institutes of Health State-of-the-Science Conference statement: preventing alzheimer disease and cognitive decline. Ann. Intern. Med. 153, 176-181. doi: 10.7326/0003-4819-153-3-201008030-00260

Dubois, B., Hampel, H., Feldman, H. H., Scheltens, P., Aisen, P., Andrieu, S., et al. (2016). Preclinical Alzheimer's disease: definition, natural history, and diagnostic criteria. Alzheimers Dement. 12, 292-323.

Eksteen, B. (2014). Advances and controversies in the pathogenesis and management of primary sclerosing cholangitis. Br. Med. Bull. 110, 89-98. doi: 10.1093/bmb/ldu008

Elliott, D. A., Kim, W. S., Jans, D. A., and Garner, B. (2007). Apoptosis induces neuronal apolipoprotein-E synthesis and localization in apoptotic bodies. Neurosci. Lett. 416, 206-210. doi: 10.1016/j.neulet.2007.02.014

Fratiglioni, L., Grut, M., Forsell, Y., Viitanen, M., Grafström, M., Holmen, K., et al. (1991). Prevalence of Alzheimer\&\#039;s disease and other dementias in an elderly urban population. Neurology 41, 1886-1892.

Germer, S., Holland, M. J., and Higuchi, R. (2000). High-throughput SNP allelefrequency determination in pooled DNA samples by kinetic PCR. Genome Res. 10, 258-266. doi: 10.1101/gr.10.2.258

Han, Z., Wang, T., Tian, R., Zhou, W., Wang, P., Ren, P., et al. (2019). BIN1 rs744373 variant shows different association with Alzheimer's disease in Caucasian and Asian populations. BMC Bioinform. 20:691. doi: 10.1186/ s12859-019-3264-9

Hanchi, H., Mottawea, W., Sebei, K., and Hammami, R. (2018). The genus Enterococcus: between probiotic potential and safety concerns-an update. Front. Microbiol. 9:1791. doi: 10.3389/fmicb.2018.01791

Harold, D., Abraham, R., Hollingworth, P., Sims, R., Gerrish, A., Hamshere, M. L., et al. (2009). Genome-wide association study identifies variants at CLU and PICALM associated with Alzheimer's disease. Nat. Genet. 41, 1088-1093.

Herz, J., and Beffert, U. (2000). Apolipoprotein E receptors: linking brain development and Alzheimer's disease. Nat. Rev. Neurosci. 1, 51-58. doi: 10. $1038 / 35036221$

Ho, L., Ono, K., Tsuji, M., Mazzola, P., Singh, R., and Pasinetti, G. M. (2018). Protective roles of intestinal microbiota derived short chain fatty acids in Alzheimer's disease-type beta-amyloid neuropathological mechanisms. Expert Rev. Neurother. 18, 83-90. doi: 10.1080/14737175.2018.1400909
Holler, C. J., Davis, P. R., Beckett, T. L., Platt, T. L., Webb, R. L., Head, E., et al. (2014). Bridging integrator 1 (BIN1) protein expression increases in the Alzheimer's disease brain and correlates with neurofibrillary tangle pathology. J. Alzheimers Dis. 42, 1221-1227. doi: 10.3233/jad- 132450

Huuskonen, J., Suuronen, T., Nuutinen, T., Kyrylenko, S., and Salminen, A. (2004). Regulation of microglial inflammatory response by sodium butyrate and shortchain fatty acids. Br. J. Pharmacol. 141, 874-880. doi: 10.1038/sj.bjp.0705682

Imhann, F., Vich Vila, A., Bonder, M. J., Fu, J., Gevers, D., Visschedijk, M. C., et al. (2018). Interplay of host genetics and gut microbiota underlying the onset and clinical presentation of inflammatory bowel disease. Gut 67, 108-119. doi: 10.1136/gutjnl-2016-312135

Jiang, H., Ling, Z., Zhang, Y., Mao, H., Ma, Z., Yin, Y., et al. (2015). Altered fecal microbiota composition in patients with major depressive disorder. Brain Behav. Immun. 48, 186-194. doi: 10.1016/j.bbi.2015.03.016

Kim, M. S., Kim, Y., Choi, H., Kim, W., Park, S., Lee, D., et al. (2020). Transfer of a healthy microbiota reduces amyloid and tau pathology in an Alzheimer's disease animal model. Gut 69, 283-294. doi: 10.1136/gutjnl-2018-317431

Lambert, J. C., Heath, S., Even, G., Campion, D., Sleegers, K., Hiltunen, M., et al. (2009). Genome-wide association study identifies variants at CLU and CR1 associated with Alzheimer's disease. Nat. Genet. 41, 1094-1099.

Lanctot, K. L., Herrmann, N., Mazzotta, P., Khan, L. R., and Ingber, N. (2004). GABAergic function in Alzheimer's disease: evidence for dysfunction and potential as a therapeutic target for the treatment of behavioural and psychological symptoms of dementia. Can. J. Psychiatry 49, 439-453. doi: 10.1177/070674370404900705

Ley, R. E., Peterson, D. A., and Gordon, J. I. (2006). Ecological and evolutionary forces shaping microbial diversity in the human intestine. Cell 124, 837-848. doi: 10.1016/j.cell.2006.02.017

Liu, P., Wu, L., Peng, G., Han, Y., Tang, R., Ge, J., et al. (2019). Altered microbiomes distinguish Alzheimer's disease from amnestic mild cognitive impairment and health in a Chinese cohort. Brain Behav. Immun. 80, 633-643. doi: 10.1016/j. bbi.2019.05.008

Mahley, R. W. (1988). Apolipoprotein E: cholesterol transport protein with expanding role in cell biology. Science 240, 622-630. doi: 10.1126/science. 3283935

Masters, C. L., Bateman, R., Blennow, K., Rowe, C. C., Sperling, R. A., and Cummings, J. L. (2015). Alzheimer's disease. Nat. Rev. Dis. Primers 1:15056.

McDonald, D., Price, M. N., Goodrich, J., Nawrocki, E. P., Desantis, T. Z., Probst, A., et al. (2012). An improved Greengenes taxonomy with explicit ranks for ecological and evolutionary analyses of bacteria and archaea. ISME J. 6, 610-618. doi: 10.1038/ismej.2011.139

McKhann, G., Drachman, D., Folstein, M., Katzman, R., Price, D., and Stadlan, E. M. (1984). Clinical diagnosis of Alzheimer's disease: report of the NINCDSADRDA Work Group under the auspices of Department of Health and Human Services Task Force on Alzheimer's Disease. Neurology 34, 939-944. doi: 10. 1212/wnl.34.7.939

Morgan, X. C., Tickle, T. L., Sokol, H., Gevers, D., Devaney, K. L., Ward, D. V., et al. (2012). Dysfunction of the intestinal microbiome in inflammatory bowel disease and treatment. Genome Biol. 13, R79.

Moustafa, A. A., Hassan, M., Hewedi, D. H., Hewedi, I., Garami, J. K., Al Ashwal, H., et al. (2018). Genetic underpinnings in Alzheimer's disease - a review. Rev. Neurosci. 29, 21-38.

Nussbaum, R. L., and Ellis, C. E. (2003). Alzheimer's disease and Parkinson's disease. N. Engl. J. Med. 348, 1356-1364.

Quigley, E. M. M. (2017). Microbiota-brain-gut axis and neurodegenerative diseases. Curr. Neurol. Neurosci. Rep. 17:94.

Russell, J. T., Roesch, L. F. W., Ordberg, M., Ilonen, J., Atkinson, M. A., Schatz, D. A., et al. (2019). Genetic risk for autoimmunity is associated with distinct changes in the human gut microbiome. Nat. Commun. 10:3621.

Saji, N., Niida, S., Murotani, K., Hisada, T., Tsuduki, T., Sugimoto, T., et al. (2019). Analysis of the relationship between the gut microbiome and dementia: a cross-sectional study conducted in Japan. Sci. Rep. 9:1008.

Schwiertz, A., Hold, G. L., Duncan, S. H., Gruhl, B., Collins, M. D., Lawson, P. A., et al. (2002). Anaerostipes caccae gen. nov., sp. nov., a new saccharolytic, acetate-utilising, butyrate-producing bacterium from human faeces. Syst. Appl. Microbiol. 25, 46-51. doi: 10.1078/0723-2020-00096

Sun, J., Xu, J., Yang, B., Chen, K., Kong, Y., Fang, N., et al. (2020). Effect of clostridium butyricum against microglia-mediated neuroinflammation in 
Alzheimer's disease via regulating gut microbiota and metabolites butyrate. Mol. Nutr. Food Res. 64:e1900636.

Tan, L., Yu, J. T., Zhang, W., Wu, Z. C., Zhang, Q., Liu, Q. Y., et al. (2013). Association of GWAS-linked loci with late-onset Alzheimer's disease in a northern Han Chinese population. Alzheimer's Dement. 9, 546-553. doi: 10. 1016/j.jalz.2012.08.007

Tran, T. T. T., Corsini, S., Kellingray, L., Hegarty, C., Le Gall, G., Narbad, A., et al. (2019). APOE genotype influences the gut microbiome structure and function in humans and mice: relevance for Alzheimer's disease pathophysiology. Faseb J. 33, 8221-8231. doi: 10.1096/fj.201900071r

Unger, M. M., Spiegel, J., Dillmann, K. U., Grundmann, D., Philippeit, H., Burmann, J., et al. (2016). Short chain fatty acids and gut microbiota differ between patients with Parkinson's disease and age-matched controls. Parkinson. Relat. Disord. 32, 66-72. doi: 10.1016/j.parkreldis.2016.08. 019

Vermunt, L., Sikkes, S. A. M., Van Den Hout, A., Handels, R., Bos, I., Van Der Flier, W. M., et al. (2019). Duration of preclinical, prodromal, and dementia stages of Alzheimer's disease in relation to age, sex, and APOE genotype. Alzheimer's Dement. 15, 888-898. doi: 10.1016/j.jalz.2019.04.001

Vogt, N. M., Kerby, R. L., Dill-Mcfarland, K. A., Harding, S. J., Merluzzi, A. P., Johnson, S. C., et al. (2017). Gut microbiome alterations in Alzheimer's disease. Sci. Rep. 7:13537.
Wijsman, E. M., Pankratz, N. D., Choi, Y., Rothstein, J. H., Faber, K. M., Cheng, R., et al. (2011). Genome-wide association of familial late-onset Alzheimer's disease replicates BIN1 and CLU and nominates CUGBP2 in interaction with APOE. PLoS Genet. 7:e1001308. doi: 10.1371/journal.pgen.100 1308

Zhang, L., Wang, Y., Xiayu, X., Shi, C., Chen, W., Song, N., et al. (2017). Altered gut microbiota in a mouse model of Alzheimer's disease. J. Alzheimers Dis. 60, 1241-1257.

Zhuang, Z. Q., Shen, L. L., Li, W. W., Fu, X., Zeng, F., Gui, L., et al. (2018). Gut microbiota is altered in patients with Alzheimer's disease. J. Alzheimers Dis. 63, 1337-1346.

Conflict of Interest: The authors declare that the research was conducted in the absence of any commercial or financial relationships that could be construed as a potential conflict of interest.

Copyright (C) $2021 \mathrm{Hou}, \mathrm{Xu}, \mathrm{Ran}, \mathrm{Luo}$ and Wang. This is an open-access article distributed under the terms of the Creative Commons Attribution License (CC BY). The use, distribution or reproduction in other forums is permitted, provided the original author(s) and the copyright owner(s) are credited and that the original publication in this journal is cited, in accordance with accepted academic practice. No use, distribution or reproduction is permitted which does not comply with these terms. 